\title{
CONVECTION IN THE MOON
}

\author{
S. K. RUNCOR N \\ Department of Geophysics and Planetary Physics, School of Physics University of Newcastle \\ upon Tyne, England
}

\begin{abstract}
It is natural to inquire whether thermal convection is occurring in the Moon through solid state creep processes. The primary evidence is the departure of the Moon from the figure of hydrostatic equilibrium, but certain difficulties in the thermal history of the Moon are eased by assuming heat transfer by convection. If convection exists in the Moon it must have a second harmonic pattern, otherwise the lunar moments of inertia would not differ.

Two important predictions of the marginal theory of convection: the existence of a core of radius 0.06- 0.3 of the lunar radius (for a second ergee harmonic) and the value of 0.4 for the ratio of the dynamical to surface ellipticities now have support, the latter from the data of the heights of the lunar surface. The former prediction is compatible with the value of the moment of inertia factor now found if the Moon's interior is 'hot'.

Further the existence of a fluid iron core 3400 m.y. ago seems required as a result of the remanent magnetization of the crystalline rocks of the maria basins inferred from the remanent magnetization of the returned Apollo samples and the fields measured by the Apollo 12 and Explorer magnetomers.
\end{abstract}

\section{The Convection Hypothesis}

Convection was first suggested in the lunar interior to explain its non-hydrostatic figure by Runcorn $(1962,1967)$. He pointed out that the discrepancy between the dynamical and the surface ellipticities of the Moon is significant and that it is naturally explained by convection because the density in the rising column is necessarily less than in the falling current. He further showed that the observed differences in the moments of inertia, apart from those corresponding to the hydrostatic model, require that the convection pattern has a second harmonic component. He also argued that if this was the main component the surface would be distorted into an ellipsoid, the long axis of which would lie in the centre of the rising or falling current.

Roberts (1965) has calculated, assuming the marginal convection theory, the ratio of the dynamical to the surface ellipticity. He used Baldwin's figure of 0.0012 for the latter and therefore he stated that this ratio (about 0.5 ) did not agree with theory and that in consequence convection in the Moon was unlikely. However as is shown in Table II the theoretical values Roberts finds depend on the boundary conditions and in his paper he favoured a free boundary for the outer surface of the convecting region. Study of continental drift, sea floor spreading and plate motions in the earth have given us a better insight into the mechanical behaviour of the solid interior of planets over long times $\left(10^{6}-10^{8} \mathrm{yr}\right)$. The crust or lithosphere behaves like a rigid body with finite strength even over such periods whereas in the mantle below, flow over such times occurs under small stress differences. This sharply contrasting mechanical behaviour results from the occurrence of a Boltzmann factor in solid state processes, including solid state creep, the large temperature gradient near the surface determining the depth at which this rather sudden change in mechanical properties occurs. 
In the Earth convection currents move the continents and plates: the boundary condition is a complex one but may be more nearly a free one than a rigid one. Each plate is large so that it is probable that the boundary condition cannot simply be a zero slip or zero stress. On the other hand there have clearly been no relative movements of parts of the lunar crust: no 'continental drift' has occurred on the Moon since the formation of the crust. Thus the appropriate boundary condition for the outer surface is certainly a rigid one, i.e. zero radial velocity gradient. Table II shows that, using the newer data, the fit of observations to the prediction of convection theory is good.

To a good approximation the ellipticity $e$ of the gravitational equipotential surface of the Moon is $\left(\frac{3}{5}\right)(C-A) / C$. Thus it can be inferred that the process which has caused the present non-equilibrium figure of the Moon does not predate the filling of the maria basins with lava but occurred some time later. This conclusion follows from Table I in which the best fit to ellipsoids of the points on the uplands and points on the

TABLE I

Axes of ellipsoids fitted to uplands, all maria, mascon maria and other maria $(\mathrm{km})$

\begin{tabular}{lllllll} 
& $\begin{array}{l}\text { Semi-axis } \\
\text { toward Earth } \\
a_{x}\end{array}$ & $\begin{array}{l}\text { Equatorial semi- } \\
\text { axis in plane of } \\
\text { sky } \\
a_{y}\end{array}$ & $\begin{array}{l}\text { Polar } \\
\text { semi-axis } \\
a_{z}\end{array}$ & $a_{x}-\left(a_{y}+a_{z}\right) / 2$ & $a_{y}-a_{z}$ & $\begin{array}{l}\text { Number } \\
\text { of points }\end{array}$ \\
\hline Uplands & 1740.2 & 1737.3 & 1736.5 & 3.3 & 0.8 & 532 \\
All maria & 1739.1 & 1736.0 & 1734.7 & 3.8 & 1.3 & 385 \\
Mascons & 1738.7 & 1734.5 & 1734.0 & 4.5 & 0.5 & 95 \\
Other maria & 1739.3 & 1736.1 & 1735.2 & 3.7 & 0.9 & 290
\end{tabular}

TABLE II

Comparison of ratios of dynamical to surface ellipticities

\begin{tabular}{lllll}
\hline Height of bulge & $\begin{array}{l}\text { Baldwin-Roberts } \\
(1949),(1965)\end{array}$ & Recent values a & Theory (Roberts, 1965) \\
\hline Dynamically & $1 \mathrm{~km}$ & $1.3 \mathrm{~km}$ & free & rigid \\
From geometrical libration & $2 \mathrm{~km}$ & $3.3 \mathrm{~km}$ & boundary & boundary \\
Ratio & 0.5 & 0.39 & 0.2006 & 0.397 \\
\hline
\end{tabular}

a Runcorn and Gray (1967); Runcorn and Shrubsall (1968); Runcorn and Hofmann, this volume, p. 22 .

maria, taken together and separated into those with mascons and those without, all yield ellipsoids of the same shape. With a reasonable accuracy the differences between the semi-axes are about the same. 


\section{Lunar Palaeomagnetism}

The magnetic studies on the Apollo 11 and 12 samples have shown that the crystalline rocks possess varying but appreciable magnetic stability, comparable with those terrestrial igneous and sedimentary rocks used in palaeomagnetic investigations. A natural remanent magnetization was found by Runcorn et al. 1970 to be possessed by the Apollo 11 crystalline rock of about $6 \cdot 10^{-6} \mathrm{emu} \mathrm{gm}^{-1}$, and it has been shown to be almost certainly acquired before it was returned to the Earth. Domains of iron were found to be responsible for the magnetic properties and these would have been likely to carry the remanent magnetization of the rock. As the blocking temperature of these particles is of the order of $600^{\circ} \mathrm{C}$, far higher than the maximum temperature reached by the rocks during the lunar day, it was concluded that the remanent magnetization was acquired when the rocks cooled from above the Curie point $\left(780^{\circ} \mathrm{C}\right)$ or soon afterwards as a result of chemical change. This clearly occurred when the rocks were part of the bedrock beneath the regolith, when they were part of the lava flows which filled Mare Tranquillitatis and Oceanus Procellarum. These lavas cooled from their melting point 3700 m.y. and 3400 m.y. ago respectively. Estimates of the field in which these lavas cooled is $1000 \gamma\left(1 \gamma=10^{-5} \mathrm{G}\right)$. This reasoning leads to the theory that the Moon possessed a field of internal origin in the first 1000 m.y. or so of its existence of about $1000 \gamma$ at the surface which it has since lost, as the field now is less than $2 \gamma$ (Runcorn et al., 1970, 1971).

This argument has been greatly strengthened by the discovery that a mean field of $36 \gamma$ (in addition to the fluctuating magnetic field of the solar wind) exists at the Apollo 12 landing site on Oceanus Procellarum. The magnetometer left there after the landing incorporated a gradiometer and as the field gradient was less than $10^{-8} \mathrm{G} \mathrm{cm}^{-1}$, Sonett concluded that the mean field must arise from an extended source of at least $1 \mathrm{~km}$ in dimension. As the regolith is only a few metres thick and as Orbiter photographs suggest that the maria basins are filled with thin and extensive lava flows lying in series, it is reasonable to infer that the mean field arises from uniformly magnetized lava flows, in which case they have intensity of about $10^{-5} \mathrm{emu}$ $\mathrm{gm}^{-1}$. But this is true only if it is assumed that different flows are magnetized in similar directions, for if lava flows covering large formations of this maria basin are each magnetized in directions more or less randomly distributed, and if the depth of the basin is small compared with its diameter, then the net fields at the surface due to each will tend to cancel out. We conclude therefore that the magnetizing field remained constant not only over the time of cooling between Curie point and blocking temperature of one flow but for most of those in the basin. On this theory the intensity of magnetization found in the Apollo samples and the mean field measured by the Apollo 12 magnetometer are consistent. This argument strongly reinforces the explanation of the remanent magnetization by a field of internal origin and not one of external origin, for the monthly rotation of the Moon requires that only the component of an external field parallel to the axis of rotation of the Moon 'can be effective'. This rules out a solar wind field even if it were reasonable to suppose it 
to be much enhanced early in the history of the solar system. Further were the geomagnetic field to be the cause, the Moon would have had to be close to the Roche limit, within 2 or 3 Earth radii, if the geomagnetic field then was about the same strength as at present. While some discussions of the early history of the Earth-Moon system entertain the possibility of such a close approach, dynamical considerations only allow the Moon to be close to the Earth for a very short time. Thus the significant difference of age between Apollo 11 and 12 crystalline rocks and the recent indications that the highlands are also magnetized, requires there to have been a field present for a considerable period in the Moon's early existence. The hypothesis that the Earth's field is responsible for this rather widespread magnetization of the lunar crust is not very attractive.

In order to explain the existence of a lunar magnetic field of internal origin in the early history of the Moon which has since disappeared it is necessary first to postulate the existence of an iron core. As is shown in Figure 1, Runcorn (1967) postulated the existence of an iron core in order that the convection in the Moon

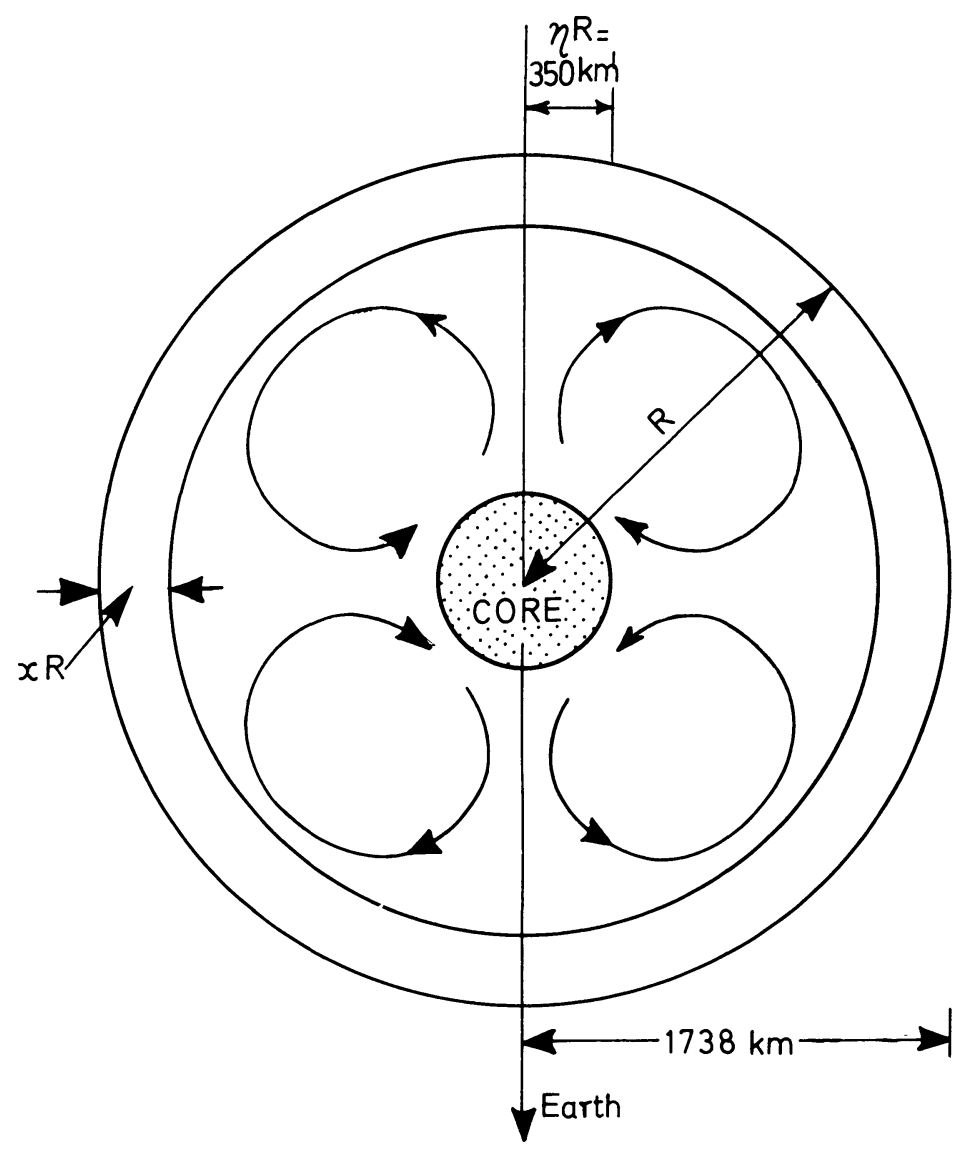

Fig. 1. 
should be a second degree harmonic pattern. According to the marginal theory of convection in spherical shells the harmonic which establishes itself depends on the ratio of the radii of the inner and outer boundary surface and also on the boundary conditions. He therefore concluded that the radius of the core would have to lie between 0.06 and 0.3 of the radius of the Moon. Because of the very small pressure gradient in the Moon, the adiabatic gradient is also small so that it seems reasonable in discussing the problem of convection in the Moon to assume that the viscosity is uniform and the results of a rather simplified convection theory can be applied. In such a core, supposed molten, convection might once have been vigorous enough to cause the magnetic field to be generated according to dynamo theory. Its later disappearance might be due to the magnetic Reynolds number falling below the critical value for dynamo action (about 10) or due to cooling of the Moon resulting in the solidification of the iron core, or due to the decrease in the angular velocity of rotation of the Moon as it recedes from the Earth. In each case the field could die away quite quickly within a period of the order of the free decay time which is about a few thousand years for a core of this size. The first alternative is rather attractive because in a small core it is not easy to make the magnetic Reynolds number great enough for dynamo action - if one supposes the radius of the iron core to be $300 \mathrm{~km}$ then the magnetic Reynolds number is about $10^{3} \times$ the velocity of the convective motions in $\mathrm{cm} \mathrm{s}^{-1}$. As the motions in the Earth's core are about $1 \mathrm{~cm} \mathrm{~s}^{-1}$, motions not much less than this would generate dynamo action. Cooling of the Moon is also suggested by the evidence that in its early history a great amount of lava was produced, whereas in recent times there appears not very much evidence for very extensive lava flows.

\section{The Existence of a Lunar Core}

Runcorn (1967) concluded from the convection theory that a small iron core exists in the Moon of between 0.06 and 0.3 of the external radius: if no core exists theory suggest that a first rather than a second harmonic convection pattern would be expected and this would neither give a bulge nor give differences in the moments of inertia. The argument that the Moon once possessed a magnetic field requires such a core, and so difierentiation must have taken place very early in lunar history and the central temperature 3000 m.y. ago must have been above M.P. of iron.

Runcorn showed that such a core was compatible with the Moon's mean density but now the question arises whether the recently determined moment of inertia factor $f=\left(C / M a^{2}\right)$ of the Moon allows the existence of a core of these dimensions. It has always been clear that a core of much greater than the above could not be allowed on density considerations: but in any case a convection pattern of higher degree than the second fails to give differences in the moments of inertia.

The thickness of the lunar crust is probably greater than the Earth's lithosphere but if the temperature gradients are comparable then, due to the low pressures in the Moon, the density in the lunar crust decreases slightly with depth in the con- 
vecting region the density is nearly constant with radius because of the very small values of the adiabatic gradient. Thus it is possible to use a simple model of the distribution of density within the Moon to test whether a lunar core is allowable.

Let $\varrho_{m}$ be the mean density of the Moon.

$\varrho_{c}$ be the density of the core.

$\varrho_{1}$ be the density of the mantle apart from the crust.

$\eta$ be the ratio of the core radius to Moon radius.

$x$ be the radio of the crust thickness to Moon radius.

$T$ be the temperature at the base of the crust.

$\alpha$ be the volume coefficient of thermal expansion of the mantle.

$$
\begin{aligned}
& \varrho_{m}=\varrho_{1}+\eta^{3}\left(\varrho_{c}-\varrho_{1}\right)+3 x \alpha T \varrho_{1} / 2 \\
& \varrho_{m}\left(1-\eta^{3} \varrho_{c} / \varrho_{m}\right)=\left(1-\eta^{3}\right) \varrho_{1}+3 x \alpha T \varrho_{1} / 2
\end{aligned}
$$

Thus

$$
f=\frac{2}{5}\left[1-\left(\eta^{3}-\eta^{5}\right)\left(\varrho_{c} / \varrho_{1}-1\right)+x \alpha T\right]
$$

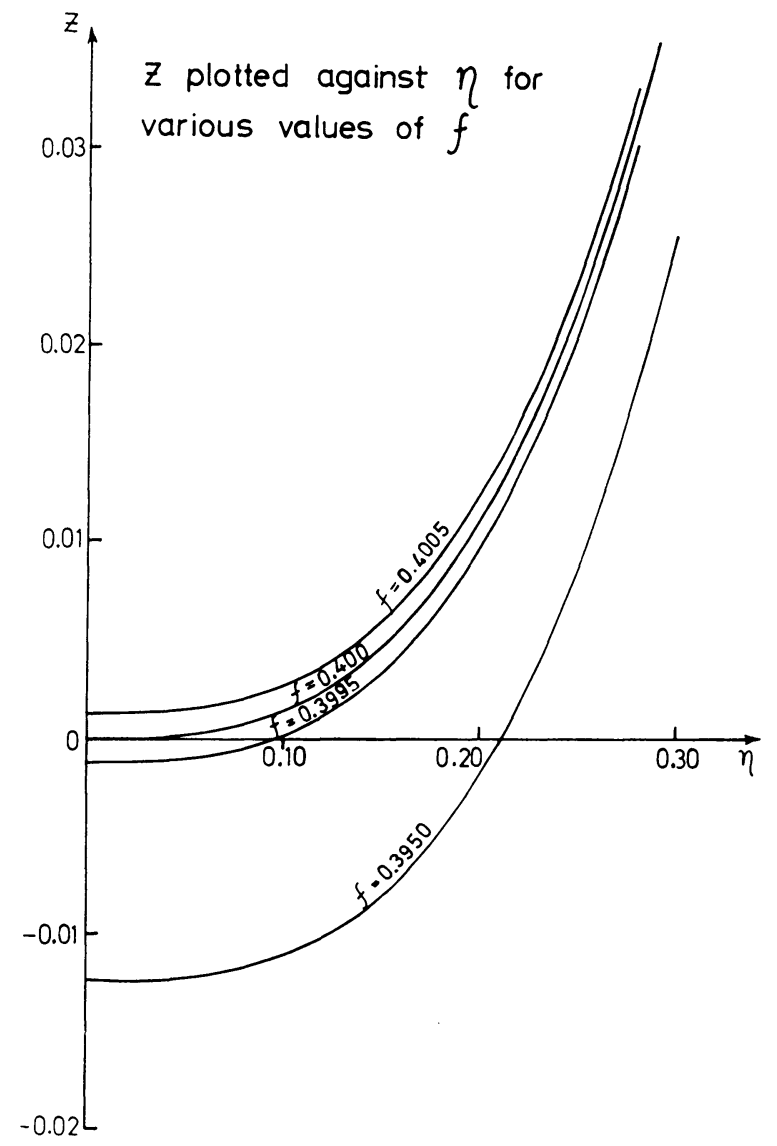

Fig. 2. 
$\varrho_{1}$ can be eliminated by combining Equations (1) and (3). Expand neglecting small terms. Thus

$$
x \alpha T=\frac{\left[\left(\varrho_{c}-\varrho_{m}\right) \eta^{3}\left(1-\eta^{2}\right) / \varrho_{m}+5 f / 2-1\right]}{\left(1+3 \varrho_{c}(5 f / 2-1) / 2\left(\varrho_{c}-\varrho_{m}\right)\right)} .
$$

Figure 2 shows $z=x \alpha T$ plotted against $\eta$ for various values of $f$ putting $\varrho_{m}=3.34$ and $\varrho_{c}=7.9$.

It seems that for the most recent value of $f$ given by Michael et al. (1970) 0.4005 , a core with $\eta=0.2$ is possible.

\section{References}

Baldwin, R. B.: 1949, The Face of the Moon, Chicago University Press.

Dyal, P., Parkin, C. W., and Sonett C. P.: 1970, Science 169, 762.

Michael, W. H.: 1970, The Moon 1, 484.

Roberts, P. H.: 1965, Mathematics 12, 128.

Runcorn, S. K.: 1962, Nature 195, 1150.

Runcorn, S. K.: 1967, Proc. Roy. Soc. A296, 240.

Runcorn, S. K. and Gray, B. M.: 1967, The Mantles of the Earth and Terrestrial Planets, John Wiley, New York.

Runcorn, S. K. and Shrubsall, M. H.: 1968, Phys. Earth Planet. Interiors 1, 317.

Runcorn, S. K., Collinson, D. W., O’Reilly, W., Stephenson, A., Greenwood, N. N., and Battey, M. H.: 1970a, Science 167, 697.

Runcorn, S. K., Collinson, D. W., O'Reilly, W., Battey, M. H., Stephenson, A., Jones, J. M., Manson, A. J., and Readman, P. W.: 1970b, Magnetic Properties of Apollo 11 Lunar Samples, Geochim. Cosmochim. Acta, Supp. 1 3, 2369.

Runcorn, S. K., Collinson, D. W., O'Reilly, W., Stephenson, A., Battey, M. H., Manson, A. J., Readman, P. W.: 1971, Proc. Roy. Soc. London A 325, 157. 